\title{
Design and characterization of inertia-activated electrical micro-switches fabricated and packaged using low-temperature photoresist molded metal-electroplating technology
}

\author{
Wei Ma ${ }^{1}$, Yitshak Zohar ${ }^{1}$ and Man Wong ${ }^{2}$ \\ ${ }^{1}$ Department of Mechanical Engineering, Hong Kong University of Science and Technology, \\ Clear Water Bay, Kowloon, Hong Kong, People's Republic of China \\ ${ }^{2}$ Department of Electrical and Electronic Engineering, Hong Kong University of Science and \\ Technology, Clear Water Bay, Kowloon, Hong Kong, People's Republic of China \\ E-mail: mawei@ust.hk,mezohar@ust.hk and eemwong@ee.ust.hk
}

Received 10 March 2003, in final form 17 June 2003

Published 14 August 2003

Online at stacks.iop.org/JMM/13/892

\begin{abstract}
Inertia-activated electrical switches have been designed and realized using a low-temperature photoresist molded metal-electroplating micro-fabrication technology compatible with processed substrates containing micro-electronic signal-processing circuits. Packaging of the switches has also been implemented using this low-temperature plating process. A simple but accurate lumped spring-mass model is developed based on analytical and numerical analyses. Predictions of the behavior of switches with a range of different designs have been verified using both drop-hammer and shaker tests. With the application of an anti-stiction hydrophobic coating, susceptibility to stiction-induced storage and operational failure has been reduced. Unencapsulated switches making over 50 million contacts have been demonstrated at room ambient.
\end{abstract}

\section{Introduction}

Electrical switches responding to changes in inertia are frequently used in toys, accessories, automotive $[1,2]$ and industrial applications. The advantages of microfabricated inertia switches are miniaturization and lowcost batch production. Furthermore, if the respective fabrication processes are compatible, switches and signalprocessing electronics can be integrated in one continuous micro-fabrication sequence. The last attribute not only simplifies device manufacturing but also enhances performance and yield. To accommodate motion, packaging solutions providing sealed and ambient-controlled cavities are required for micro-systems containing mechanical components [3]. Such solutions are often more complicated and expensive than those for the packaging of pure electronic devices.
The present work was motivated by the desire to develop a sufficiently general low-temperature fabrication process that can be used to modularly fabricate and package a variety of metallic, hence conducting, micro-mechanical structures integrated on processed active substrates containing microelectronic signal-processing circuits. Inertia-activated microswitch was used as a demonstration vehicle.

Photoresist molded [4] low-temperature metalelectroplating [5] technology, allowing the formation of films with thickness (hence structural height) not readily achievable using conventional surface micro-fabrication techniques, has been reported by Engelmann [6]. The processes and the materials employed in low-temperature electroplating of metals are largely compatible with active substrates. Consequently, if other issues such as the proper selection of sacrificial layers can be resolved, metal electroplating can potentially be applied to modularly 


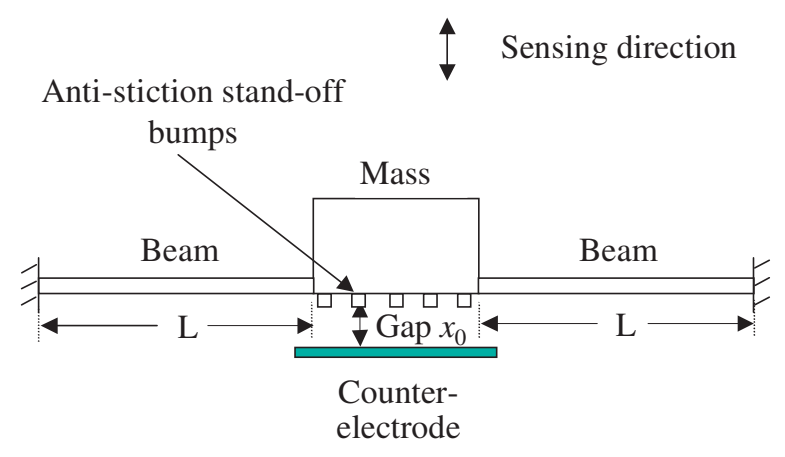

(a)

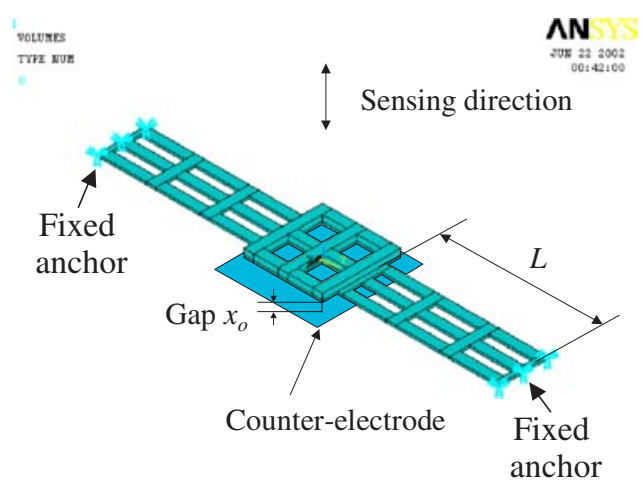

(b)

Figure 1. Schematic diagram of a vertically driven inertia micro-switch. (a) Cross-sectional view and $(b)$ finite-element model.

integrate a range of micro-mechanical structures on processed active substrates.

Multiple cycles of photoresist molded gold ( $\mathrm{Au})$ plating combined with aluminum (Al) sacrificial layers are presently applied to the fabrication of inertia-activated micro-switches with discriminating sensitivities to inertia changes [7, 8]. Unlike the laterally driven switches reported in [8], those presently reported are vertically driven. These switches are realized using a process that allows a more compact switch design and the flexibility of using different structural heights for the mass and spring.

A wafer-level packaging technique including integrated electrical access has also been developed [8], based on the electroplating of a metallic 'sealant' on a separate 'lid wafer' and its subsequent bonding to a 'device wafer' containing mechanical and other components [9]. The height of the cavity is determined by the thickness of the sealant. The countersealing metal (CSM) on the device wafer has been seamlessly integrated into the device fabrication flow, using a separate but non-interfering electroplating process. A low resistance $(\sim 12 \Omega)$ electrical access is provided to the sealed devices.

The design process was aided by a simple but accurate lumped spring-mass model based on analytical and numerical analyses. Predictions of the behavior of the switches with various mechanical designs have been verified using both drophammer and shaker tests. A vapor-phase deposition technique has been employed to apply a hydrophobic monolayer coating to reduce susceptibility to stiction-induced storage and operational failure. Unencapsulated switches operating in room ambient making over 50 million contacts have been demonstrated.

\section{Design and simulation}

A spring-mass-spring bridge type design was adopted for the switches. With the switch at rest, the proof mass as one electrode is separated from the corresponding counterelectrode by a gap of $x_{0}$. The sensitive axis is normal to the surface of the substrate (figure 1).

Assuming the system could be represented by a finite mass $m$ attached to a massless spring with a force constant $k$ driven by a force $F$, the corresponding governing equation would be

$$
m \ddot{x}=-\beta \dot{x}-k x+F,
$$

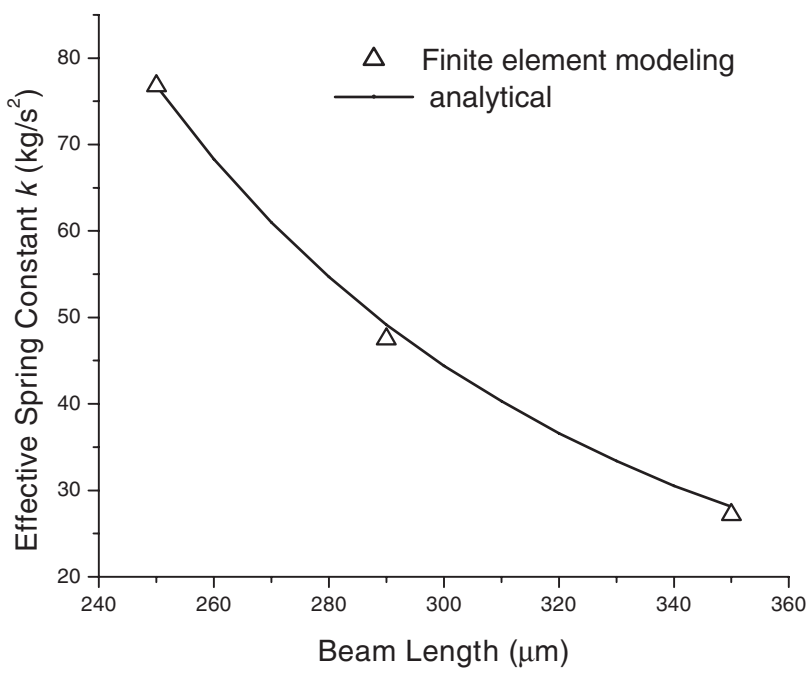

Figure 2. A comparison between the analytical and simulated effective spring constants.

where $\beta$ is a first order damping coefficient. The static deflection $x_{\mathrm{s}}$ under a static acceleration $a_{\mathrm{s}}$ is

$$
x_{\mathrm{s}}=\frac{m}{k} a_{\mathrm{s}} \text {. }
$$

The amplitude of a sinusoidal deflection $x_{\mathrm{d}}$ under a sinusoidal acceleration with amplitude $a_{\mathrm{d}}$ and frequency $\omega$ is [10]

$$
x_{\mathrm{d}}=\frac{a_{\mathrm{d}}}{\sqrt{\left(\frac{k}{m}-\omega^{2}\right)^{2}+\left(\frac{\beta \omega}{m}\right)^{2}}} .
$$

For a contact to be made, $x_{\mathrm{s}}$ and $x_{\mathrm{d}}$ must be greater than or equal to $x_{0}$. The minimum static or dynamic accelerations required to activate a switch are defined to be the corresponding 'threshold' accelerations.

In the case of an 'impulse' force, the proof mass is instantaneously accelerated and a 'threshold' velocity $v_{\mathrm{t}}$ can be defined using the energy conservation equation derivable from equation (1):

$$
v_{t}=\sqrt{\frac{k}{m}} x_{0},
$$

where damping has been ignored. 


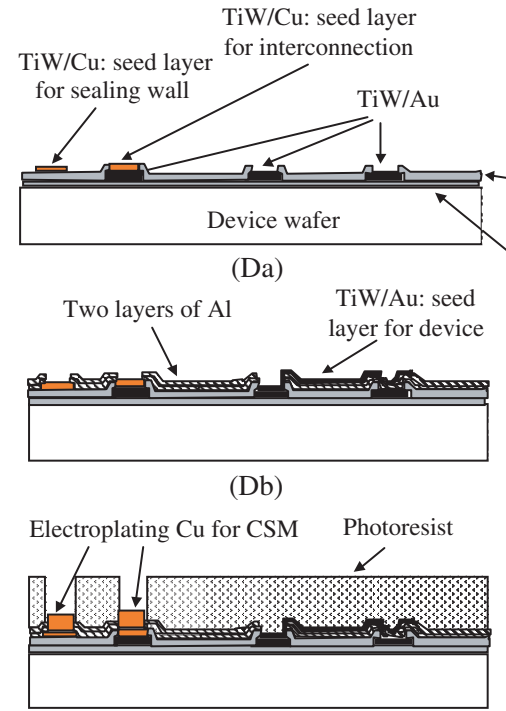

(Dc)

Electroplating $\mathrm{Au}$ for the beam of the switch

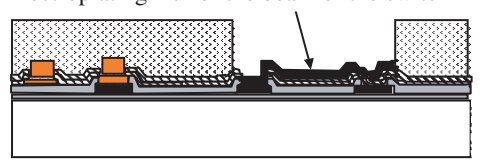

(Dd)

Electroplating $\mathrm{Au}$ for the mass of the switch

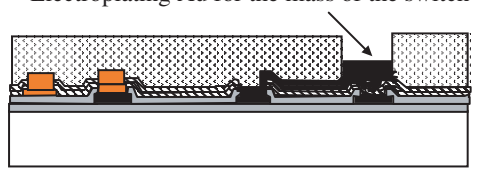

(De)

Released devices

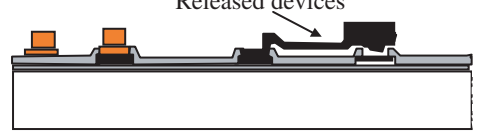

(Df)

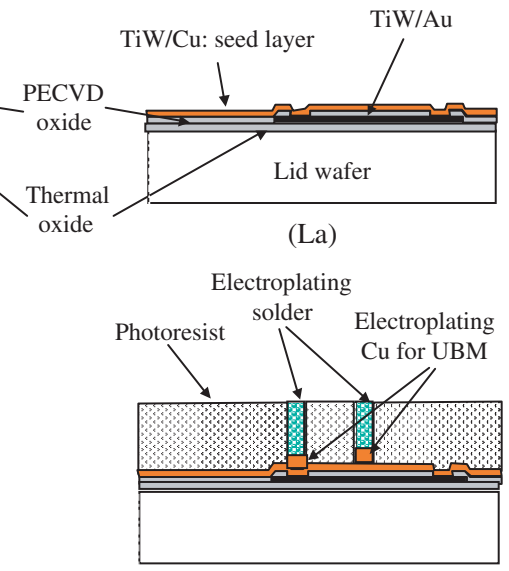

(Lb)

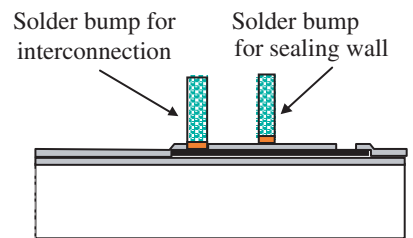

(Lc)

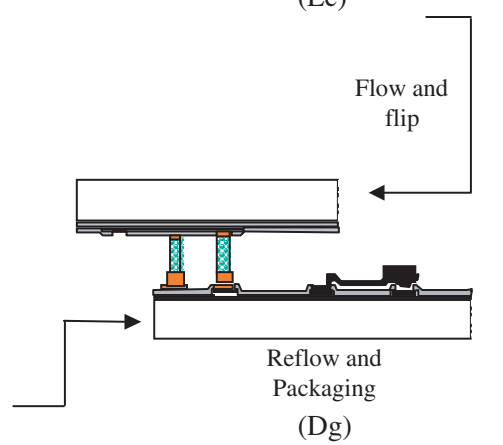

(Dg)

Figure 3. Process flow for the fabrication and packaging of an inertia micro-switch.

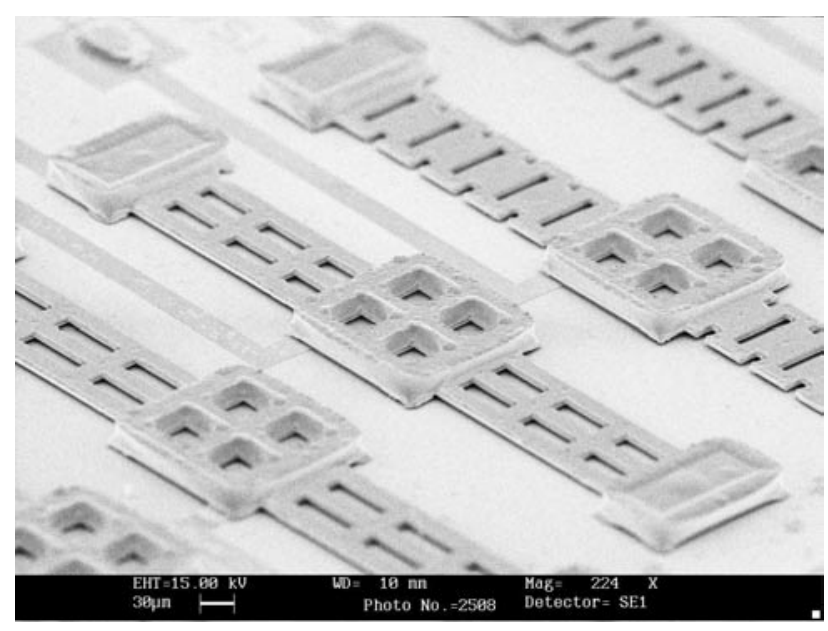

Figure 4. SEM micrograph of fabricated switches.

The selected design (figure 1) allows the switch to be modeled as a double-clamped massless beam with a proof mass $m$ concentrated at the center. The effective spring constant $k$

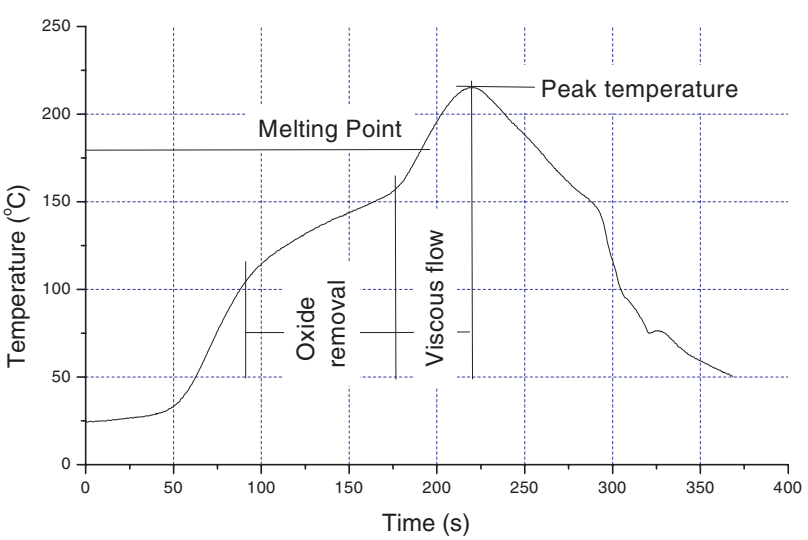

Figure 5. Temperature profile during solder flow and re-flow. can be written as [11]:

$$
k=\frac{192 E I}{L^{3}},
$$

where $E$ is Young's modulus, $I$ is the effective moment of inertia of the beam in the sensing direction and $L$ is the beam length. 


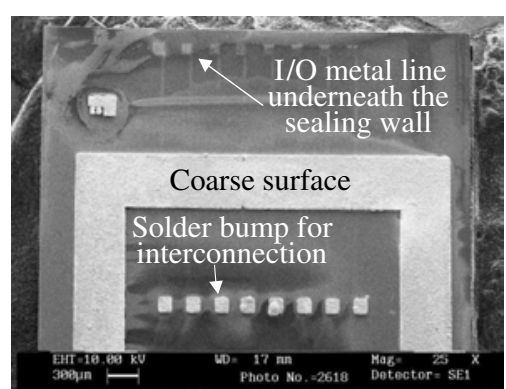

(a)

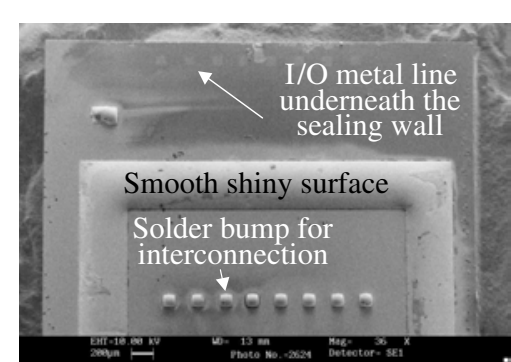

(b)

Figure 6. Surface finish of solder on the lid die $(a)$ before and $(b)$ after solder flow.

Extensive simulations were performed using ANSYS 5.7, a commercial finite-element modeling (FEM) software package. Good agreement (figure 2) is obtained between the analytically derived (equation (5)) and numerically extracted $k$ values. It is also verified that the analytical model agrees well with the simulated fundamental vibration mode of the structure in terms of such parameters as the resonant frequency, the stress on critical points and transient displacement. A series of devices with different threshold velocities are designed based on equation (4).

\section{Switch fabrication and packaging}

The switches, together with their CSM for packaging, were realized using a nine-mask process. Electroplated $\mathrm{Au}$ was selected as the structural material for its stability and low resistance. The fabrication sequence is schematically shown in figure 3.

Silicon wafers covered with $0.2 \mu \mathrm{m}$ insulating oxide were used as the starting substrates. $30 \mathrm{~nm}$ titanium (Ti) adhesion promoter and $200 \mathrm{~nm} \mathrm{Au}$ were sputtered and patterned to form the electrical access and an initial interconnect pattern (figure 3Da). Low-temperature silicon oxide was subsequently deposited and patterned to form a top insulation layer.

On the device wafer, a titanium:tungsten/copper $(\mathrm{TiW} / \mathrm{Cu})$ stacked layer was sputtered and patterned as the seed layer for the plating of the CSM for the vertical interconnections and the sealing wall. $\mathrm{Al}$ was chosen as the sacrificial layer for its low temperature of formation, high electrical conductivity and excellent etch selectivity to both the Au structural material and the underlying oxide insulator. A first layer of $0.5 \mu \mathrm{m} \mathrm{Al}$ was sputtered and patterned to form an array of holes for the definition of anti-stiction stand-off bumps over the contact area of the mass electrode. A second layer of $\mathrm{Al}$ was sputtered to form the sacrificial layers. Its $2 \mu \mathrm{m}$ thickness defined the electrode gap spacing $x_{\mathrm{o}}$. The Al layers also formed a continuous conductive base to provide the current path during electroplating on the otherwise isolated seed layers. The anchor areas through which the beams would be clamped and the electrodes would be electrically connected, together with the CSM area for the sealing wall and the vertical interconnections, were opened. A second $30 \mathrm{~nm} / 100 \mathrm{~nm}$ TiW/Au seed layer was deposited and patterned using a liftoff technique (figure 3Db).

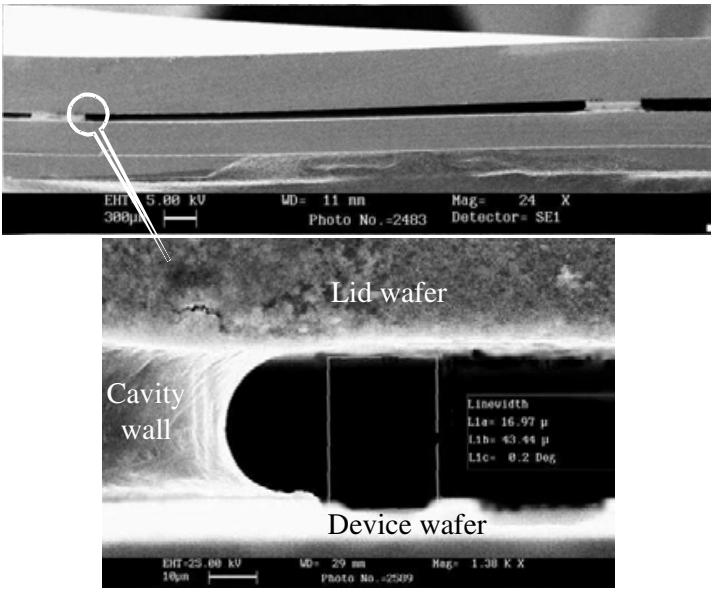

Figure 7. Cross-sectional SEM micrograph of a pair of bonded device and lid wafers.

Clariant AZ UTP92, a high viscosity and high transparency photoresist, spun using a conventional spinner, was used to form the plating molds. A thickness up to $30 \mu \mathrm{m}$ could be spun at $700 \mathrm{rpm}$. A two-step hot-plate pre-baking to minimize bubble formation was done, first at $50{ }^{\circ} \mathrm{C}$ for $5 \mathrm{~min}$ and then at $110^{\circ} \mathrm{C}$ for $3 \mathrm{~min}$. Exposure was done in a contact aligner, at an energy density of $400 \mathrm{~J} \mathrm{~cm}^{-2}$ for a $30 \mu \mathrm{m}$ thick resist. The resist was developed by immersion in an alkaline developer ( $\left.\mathrm{AZ} 400 \mathrm{~K}: \mathrm{H}_{2} \mathrm{O}=1: 2\right)$. The resolution limit was about $8 \mu \mathrm{m}$ for a $30 \mu \mathrm{m}$ thick resist. A two-step hot-plate post-bake to minimize deformation due to resist flow was done, first at $50{ }^{\circ} \mathrm{C}$ for $10 \mathrm{~min}$ to stabilize the sidewall and then at $90{ }^{\circ} \mathrm{C}$ for $10 \mathrm{~min}$ to harden the resist.

$\mathrm{Cu}$ was first electroplated at a current density of $40 \mathrm{~mA} \mathrm{~cm}{ }^{-2}$ at room temperature to form the $5 \mu \mathrm{m}$ thick CSM (figure 3Dc). The plating solution was Copper Gleam 2001 from LeaRonal. Commercially available Neutronex 309 solution at $50{ }^{\circ} \mathrm{C}$ was used for Au electroplating to form the switches. A current density of $2 \mathrm{~mA} \mathrm{~cm}^{-2}$ was chosen to maintain a fine grain texture and to minimize the intrinsic film stress. The plating rate was $\sim 3.6 \mu \mathrm{m} \mathrm{h}^{-1}$. The uniformity across a $100 \mathrm{~mm}$ wafer is within $1 \mu \mathrm{m}$ for a $25 \mu \mathrm{m}$ deposition.

A first photoresist mold was used to define the $5 \mu \mathrm{m}$ thick beam spring (figure 3Dd) of a switch. A second photoresist mold was used to define a $20 \mu \mathrm{m}$ thick Au plating, forming 


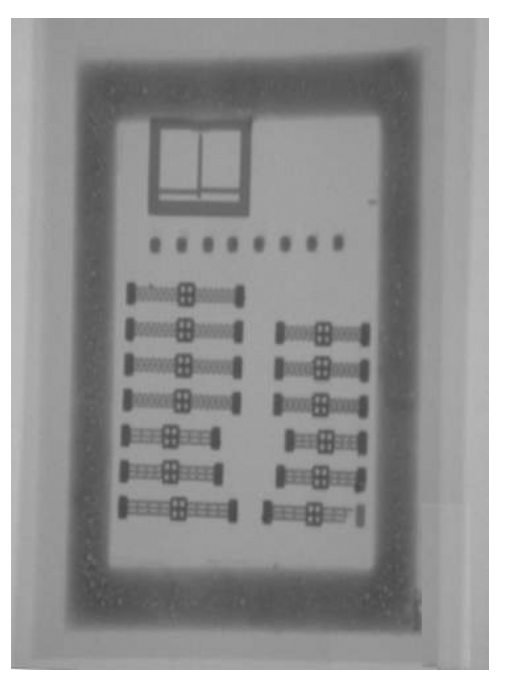

(a)

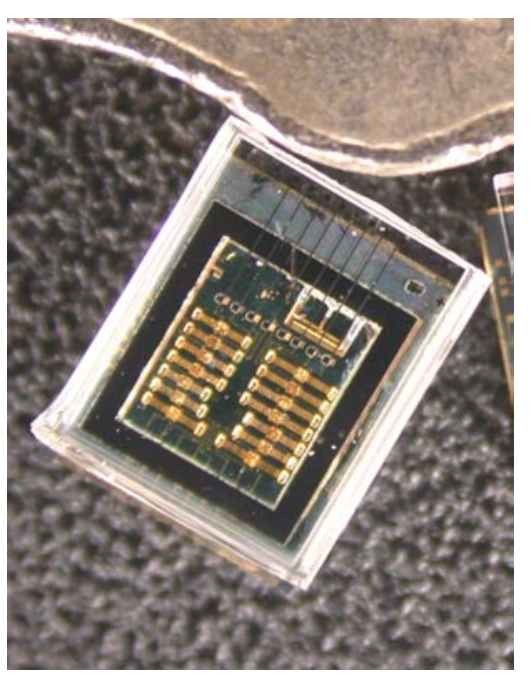

(b)

Figure 8. (a) X-ray micrograph of a packaged die with an opaque lid. (b) Photograph of packaged die with transparent lid.

the mass of the switch (figure 3De). After the removal of the photoresist mold, the Al sacrificial layer was etched in $1 \mathrm{~h}$ in a phosphoric acid based $\mathrm{Al}$ etchant to free the structures (figure 3Df).

Stiction, to which this kind of vertically driven switch is particularly susceptible, has to be minimized during the drying process after the sacrificial layer etch and deionized water rinse. This was accomplished by displacing any residual water by soaking in isopropyl alcohol for $1 \mathrm{~h}$. The switches were dried by allowing the residual alcohol to evaporate in room ambient. To reduce susceptibility to stiction during operation, the switches were placed on a $110{ }^{\circ} \mathrm{C}$ hot-plate and exposed to hexamethyldisilazane (HMDS) vapor for $90 \mathrm{~s}$. A thin layer of hydrophobic HMDS was formed on exposed Au surfaces. A scanning electron micrograph (SEM) of the finished devices is shown in figure 4 .

After the sputter deposition of a $\mathrm{TiW} / \mathrm{Cu}$ seed layer (figure 3La), and the electroplating of a 5-7 $\mu \mathrm{m} \mathrm{Cu}$ underbump metal (UBM), eutectic tin:lead $\left(\mathrm{Sn}_{63}: \mathrm{Pb}_{37}\right)$ solder, which formed both the vertical interconnections and the sealing wall, was electroplated (figure 3Lb) on a silicon or glass lid wafer. The height of the wall was adjusted to be higher than that of the switches. After removal of the photoresist plating mold, any exposed $\mathrm{TiW} / \mathrm{Cu}$ seed layer was removed in ShipleyRonatch PS mixed with $\mathrm{H}_{2} \mathrm{SO}_{4}$ and $\mathrm{H}_{2} \mathrm{O}_{2}$ at $40{ }^{\circ} \mathrm{C}$ respectively (figure 3Lc). A volatile flux (Heraeus Wave Flux) requiring no post-clean was brushed on the solder. Solder flow was accomplished in an oven using the time-temperature profile shown in figure 5. A relatively long soak between 100 and $150{ }^{\circ} \mathrm{C}$ was used to break down the surface oxide. Intermetallic compound was formed at the interface of the solder and its UBM when the temperature was raised beyond the eutectic melting point $\left(\sim 183^{\circ} \mathrm{C}\right)$ of the solder. The peak temperature during the solder flow was $220^{\circ} \mathrm{C}$. The surface of the solder became smooth and shiny after the flow, as shown in figure 6 .

The device and the lid wafers were subsequently diced and pairs of device and lid dice were bonded. Alignment to the $\mathrm{CBM}$ on a device die containing released inertia

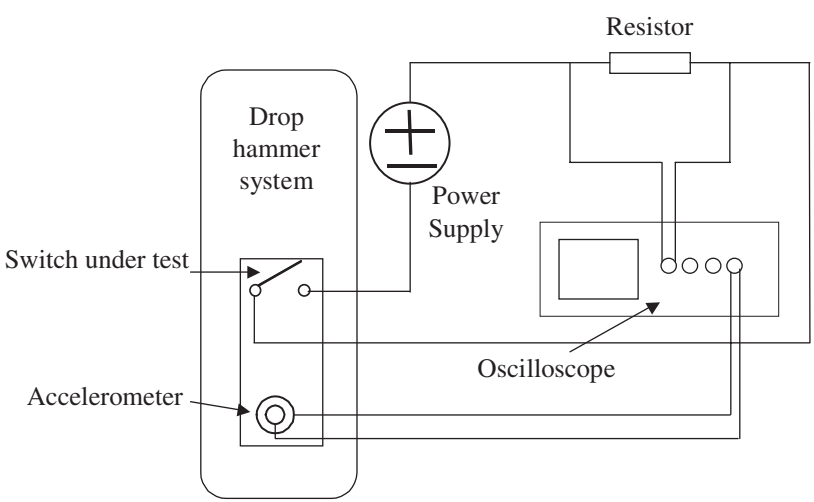

Figure 9. A schematic of the electrical setup for the drop-hammer test.

micro-switches was done on a micro-placer (MP-2000 series). Bonding (figure $3 \mathrm{Dg}$ ) was performed in an oven using the same temperature profile as shown in figure 5. No additional force was applied during the bonding process. The height of the cavity (figure 7) is largely defined by that of the solder. The ability to bond was not affected by the $200-500 \mathrm{~nm}$ topography of the electrical access. A pair of packaged dies is shown in figure 8.

\section{Testing and characterization}

A drop-hammer setup was used to determine the threshold velocity $v_{\mathrm{t}}$ of the switches. A switch-under-test was connected to a $3 \mathrm{~V}$ power supply through a $1 \mathrm{k} \Omega$ current-limiting resistor. The signal was detected using an oscilloscope. A schematic of the electrical setup is shown in figure 9. The free-falling hammer was released from a pre-determined height $H$. An impulse response was recorded by the switch when a contact was made. The response of the switch, together with that of an accelerometer attached to the drop-hammer, is shown in figure 10. The accelerometer is assumed to record instantaneously the moment of the impact. Therefore, the 


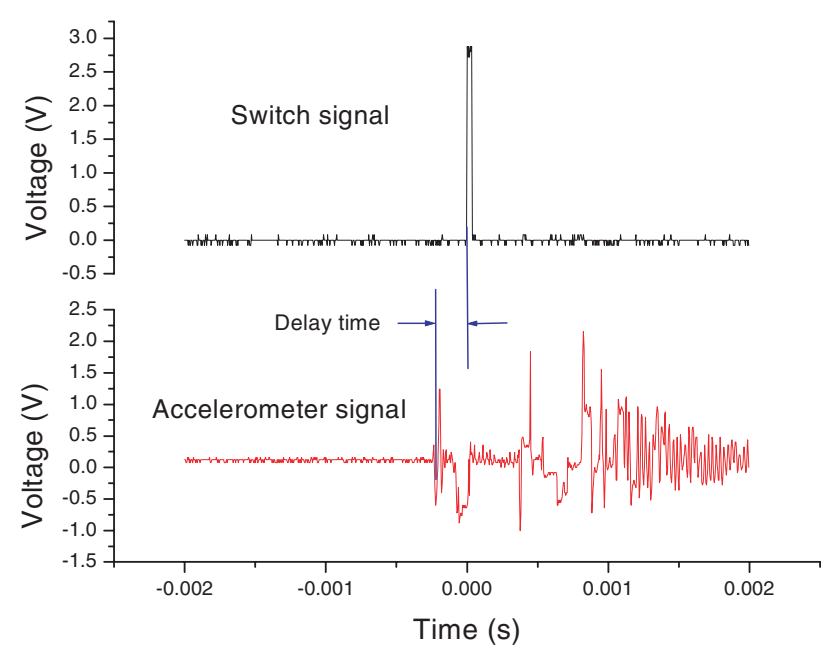

Figure 10. Response signals of a switch and an acceleromer.

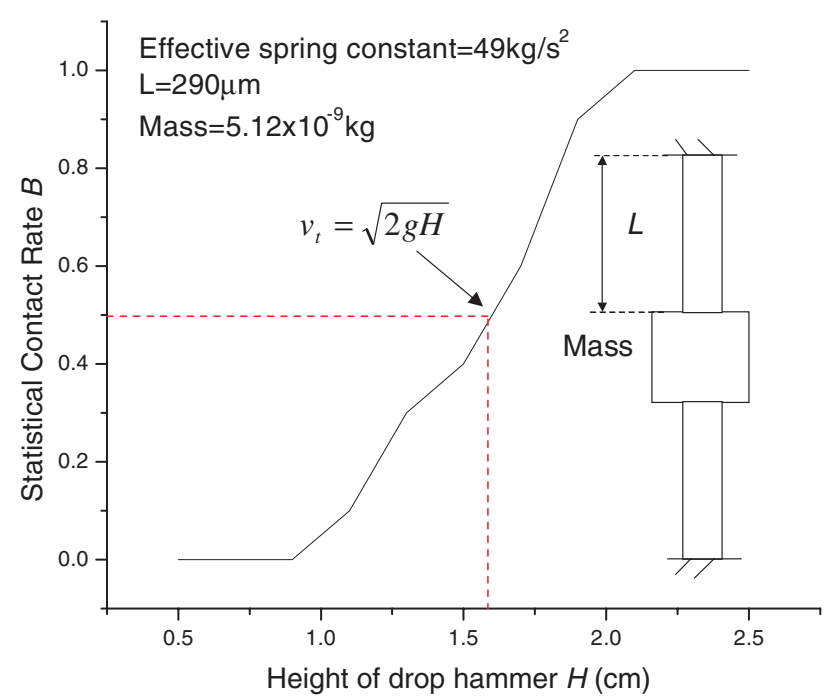

Figure 11. Dependence of the contact rate $B$ on hammer height $H$.

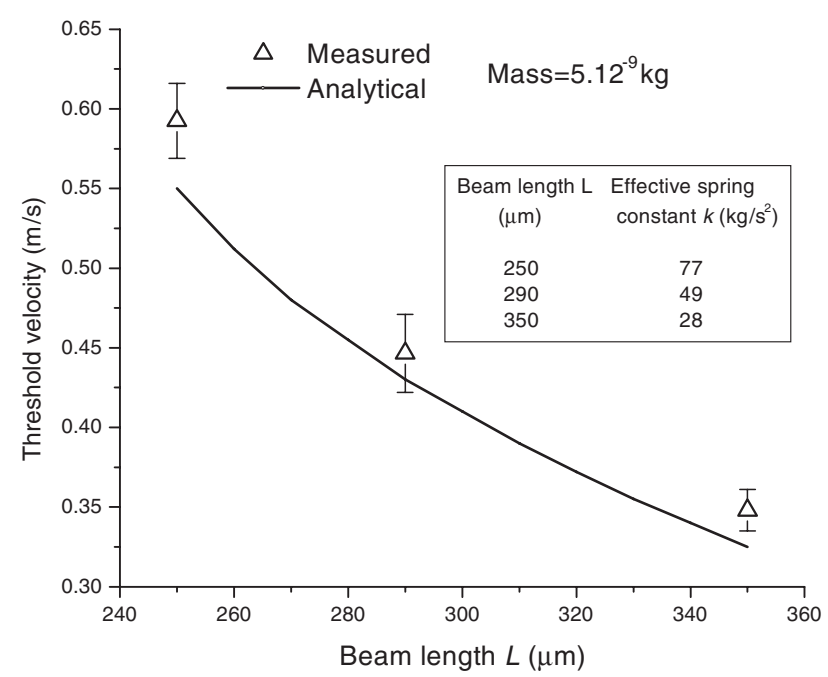

Figure 12. Dependence of threshold velocity $v_{\mathrm{t}}$ on different switch designs.
POLUME
TYPE NUM

$\mathbf{\Lambda N}$

TYPE MUS

HAR 192002

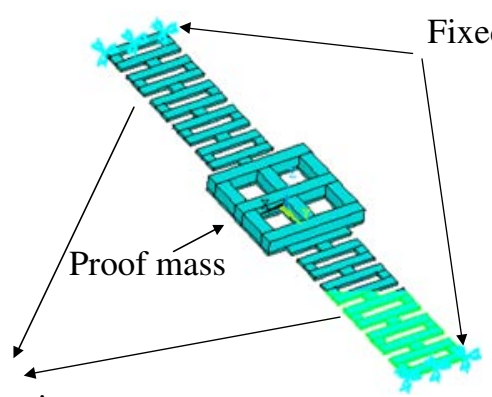

Loop-spring

Figure 13. Switch design with stress-relieved loop-springs.

Top: Switch response

Bottom: Accelerometer response

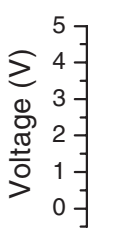

(a) $\mathrm{f}=5.55 \mathrm{kHz} \quad B=0.3$

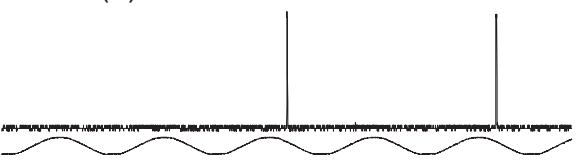

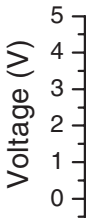

(b) $f=5.80 \mathrm{kHz} \quad B=0.7$

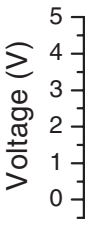

(c) $\mathrm{f}=6.18 \mathrm{kHz} \quad B=1$
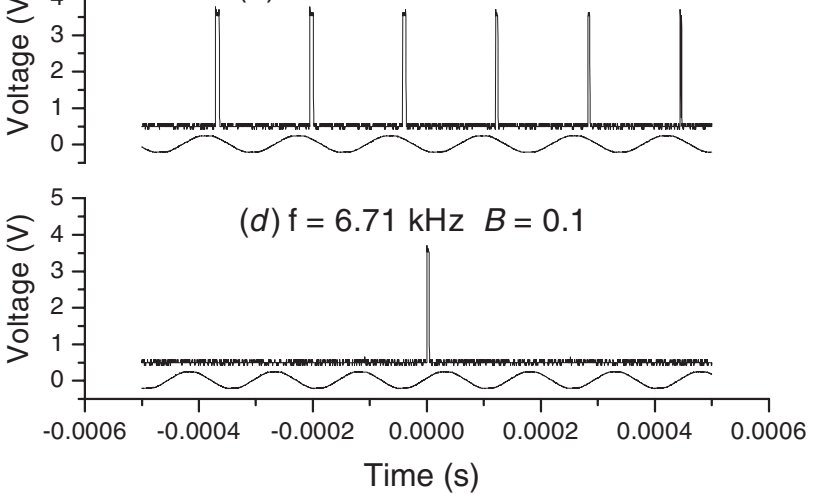

Figure 14. Dependence of the switch response on shaker frequency $f$.

delay between the impulse response of the switch and the initial signal of the accelerometer is the time taken by the mass-electrode to travel the gap $x_{\mathrm{o}}$. It can be inferred from the small difference between the $3 \mathrm{~V}$ power supply and the peak response voltage that the total resistance (including contact and leads) is $\sim 30 \Omega$. For any given $H$, the test was repeated a number of times to obtain a statistical contact rate $B$, defined to be the ratio of the number of contacts made to the number of drops. The dependence of $B$ on $H$ is plotted in figure 11 . It can be seen that $B$ increased gradually from 0 to 1 when $H$ 


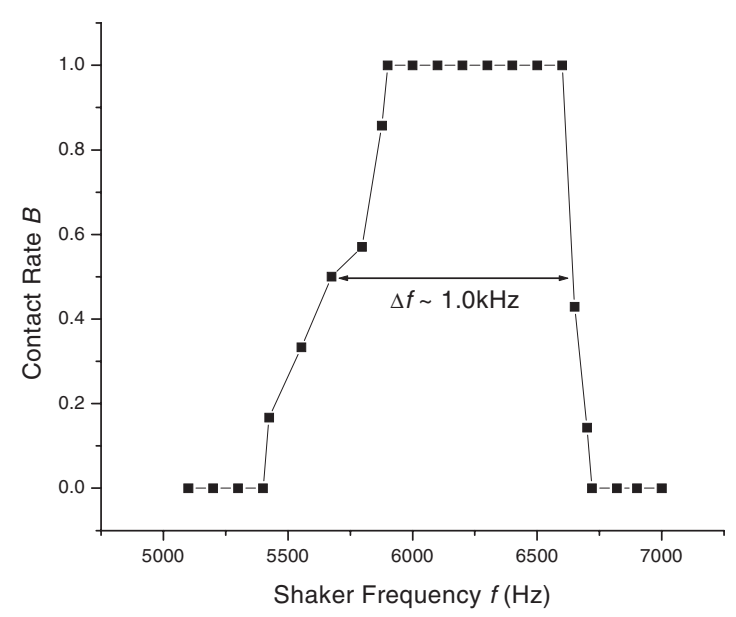

Figure 15. Dependence of the contact rate $B$ on shaker frequency $f$.

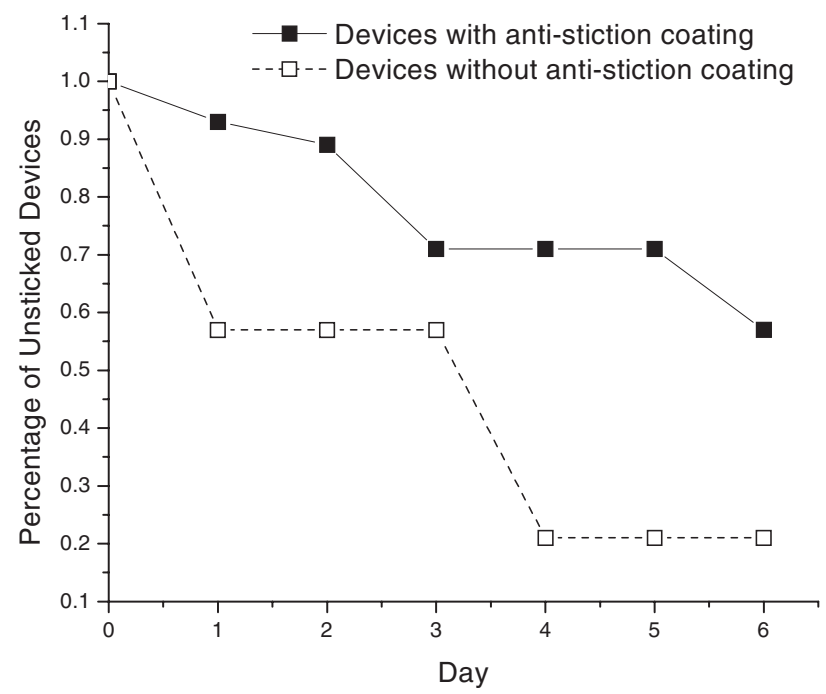

Figure 16. Comparison of the storage lifetime of switches with and without the anti-stiction coating.

was raised from $\sim 1 \mathrm{~cm}$ to $\sim 2 \mathrm{~cm}$. The velocity of the hammer immediately before the impact corresponding to $H$ at $B=0.5$ is defined to be $v_{\mathrm{t}}$.
A comparison between the measured and the analytically derived $v_{\mathrm{t}}$ is shown in figure 12. Though the general agreement is good, it is clear that the measured $v_{\mathrm{t}}$ is consistently higher than the analytically derived $v_{\mathrm{t}}$. Since variations in the dimensions of spring beams and mass have been incorporated in the simulations, the most likely cause of the deviation is residual stress in the Au structural film. Although a low plating current density was used to reduce the residual stress, a non-zero residual stress remained. This 'stiffened' the beams and led to a higher effective spring constants $k$, thus also to a consistently higher $v_{\mathrm{t}}$. The way to relax the remaining residual stress, hence to allow a more compact switch layout, is to use a loop-spring design, as shown in figure 13.

The resonant frequency was measured by attaching a switch and an accelerometer to a shaker. Under a sinusoidal excitation, an equivalent contact rate $B$ was defined to be the average number of contacts made per period of oscillation (figure14). The dependence of $B$ on $f$ is shown in figure 15, from which an approximate resonant frequency of $6.2 \mathrm{kHz}$ is obtained. This is within $7 \%$ of the analytically predicted value for this design. A 'bandwidth' of $1.0 \mathrm{kHz}$ can be estimated using the difference between the two frequencies at $B=0.5$. The small $Q$-factor clearly indicates the switch suffers from a non-trivial squeezed-film damping effect.

Susceptibility to stiction at room ambient was compared and shown in figure 16 for unpackaged switches with and without a hydrophobic monolayer coating. Switches without the coating showed rapid stiction-induced degradation over time. With the coating, storage reliability against stiction was significantly improved. The operational stability of the switches was poor without the coating, failing after making a small number (below four million) of contacts. With the coating, storage reliability against stiction was significantly improved. Furthermore, continuous operation over 50 million contact cycles has been demonstrated, with no observable changes in the mechanical or electrical behavior of the switches.

The resistance of the vertical interconnections in the packaged pair was measured using a cross-bridge structure that is schematically shown in figure 17. A test current was forced between pads 1 and A. The corresponding voltage was measured between pads 2 and B. A low resistance of $\sim 12 \Omega$ was measured.

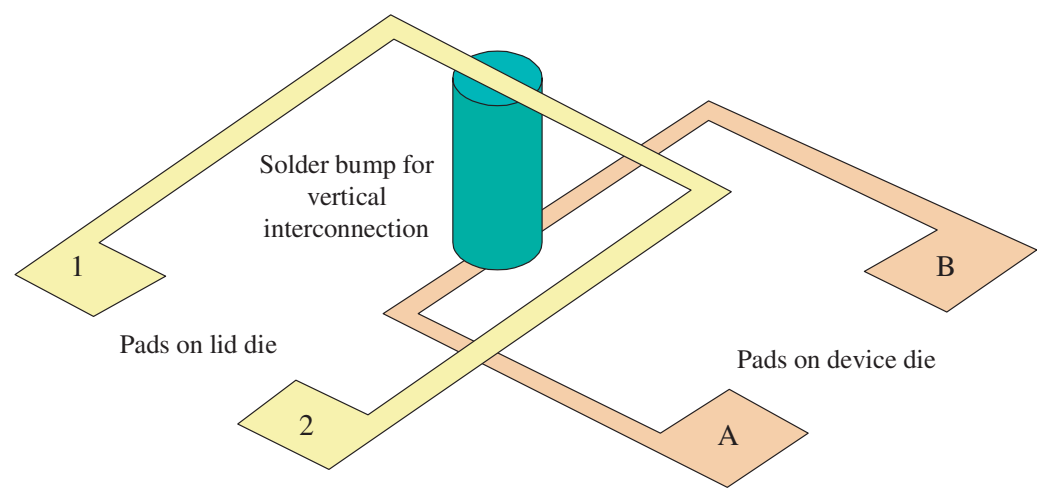

Figure 17. Schematic diagram of the cross-bridge structure for interconnection resistance measurement. 


\section{Conclusions}

The micro-inertia switches have been designed and fabricated using a low-temperature, photoresist molded, metal-electroplating technology. Their wafer-level packaging in sealed cavities was also implemented using the same metal-electroplating technology. The height of a switch and that of its cavity are independently controlled. The scheme also provides leads for low-resistance electrical access to the sealed devices. A simple design methodology is developed based on analytical and detailed numerical analyses. Switch designs have been verified by subjecting fabricated switches to drophammer and shaker tests. With an anti-stiction HMDS coating, unencapsulated switches making over 50 million contacts have been demonstrated at room ambient.

\section{Acknowledgments}

The support of China Resources Semiconductor Co., Ltd and the Institute of Integrated Micros-Systems of the Hong Kong University of Science and Technology is gratefully acknowledged.

\section{References}

[1] Tønnesen T, Lüdtke O, Noetzel J, Binder J and Mader G 1997 Simulation, design and fabrication of electroplated acceleration switches J. Micromech. Microeng. 7 237-9

[2] Michaelis S, Timme H-J, Wycisk M and Binder J 2000 Additive electroplating technology as a post-CMOS process for the production of MEMS acceleration-threshold switches for transportation application J. Micromech. Microeng. 10 120-3

[3] Gilleo K 2001 Overview of new packages, materials and processes Int. Symp. on Advanced Packaging Materials pp 1-5

[4] Loechel B 2000 Thick layer resists for surface micro-machining J. Micromech. Microeng. 10 $108-15$

[5] Ruythooren W, Attenborough K, Beerten S, Merken P, Fransaer J, Beyne E, van Hoof C, de Boeck $\mathrm{H}$ and Celis J P 2000 Electrodepositon for the synthesis of microsystems J. Micromech. Microeng. 10 101-7

[6] Engelmann G, Ehrmann O, Simon J and Reichi H 1992 Fabrication of high depth-to-width aspect ratio microstructures MEMS '92 pp 93-8

[7] Ma W, Zohar Y and Wong M 2003 Design and characterization of micro-inertia switches fabricated using low-temperature metal-electroplating technology MEMS Components and Applications for Industry, Automobiles, Aerospace, and Communication II, Proc. SPIE (San Jose, CA, 28-29 Jan. 2003) pp 214-21

[8] Ma W, Li G, Zohar Y and Wong M 2003 Fabrication and packaging of inertia micro-switch using metal-electroplating technology MEMS 2003. 14th IEEE Int. Conf. (Kyoto, Japan, 20-23 Jan. 2003) pp 610-3

[9] Tilmans H A C, Van de Peer M D J and Beyne E 2000 The indent reflow sealing (IRS) technique-a method for the fabrication of sealed cavities for MEMS devices J. Microelectromech. Syst. 9 206-16

[10] Weaver W, Timoshenko S and Young D H 1990 Vibration Problems in Engineering 5th edn (New York: Wiley)

[11] Timoshenko S 1958 Strength of Materials (New York: Van Nostrand-Reinhold) 